\title{
Projected Spin Networks for Lorentz connection: Linking Spin Foams and Loop Gravity
}

\author{
Etera R Livine \\ Centre de Physique Théorique, \\ Luminy, Case 907, Marseille, France \\ livine@cpt.univ-mrs.fr
}

October 24, 2018

\begin{abstract}
In the search for a covariant formulation for Loop Quantum Gravity, spin foams have arised as the corresponding discrete space-time structure and, among the different models, the Barrett-Crane model seems the most promising. Here, we study its boundary states and introduce cylindrical functions on both the Lorentz connection and the time normal to the studied hypersurface. We call them projected cylindrical functions and we explain how they would naturally arise in a covariant formulation of Loop Quantum Gravity.
\end{abstract}

\section{Introduction}

Constructing an explicitely covariant formulation of Loop Quantum Gravity (see [24] for a review) seems a natural step towards solving some issues such as the physical role of the Immirzi parameter, which is likely to disappear in a fully covariant approach [16], or the exact form of the hamiltonian constraint. When studying the observables of the theory, the main difficulty is to deal with a non-compact gauge group, the Lorentz group. Although spin networks states have been defined for non-compact groups [1], much still needs to be done before getting a full consistent theory. In the present paper, another type of spin networks is defined which can be used for non-compact gauge groups but which only have a compact gauge symmetry. To understand where they come from, we should review the different approaches to understanding the physical content of the (generalised) Palatini action [21] :

$$
S=\frac{1}{2} \int \epsilon_{\alpha \beta \gamma \delta} e^{\alpha} \wedge e^{\beta} \wedge\left(F^{\gamma \delta}+\frac{1}{\gamma} \star F^{\gamma \delta}\right)
$$

where $\alpha, \ldots$ are internal Lorentz indices, $e$ is the tetrad field, $F$ is the curvature of the space-time connection, $\star$ is the Hodge operator $\star F^{\alpha \beta}=\frac{1}{2} \epsilon^{\alpha \beta}{ }_{\gamma \delta} F^{\gamma \delta}$ and $\gamma$ is the Immirzi parameter. The main problem occuring in the canonical analysis of this action is the second class constraints. There have been four main paths explored in order to deal with them.

- Solving the constraints: Loop Quantum Gravity

Holst showed it is possible to derive the canonical structure of loop gravity with Immirzi parameter by considering a generalised Palatini action which is related to the original by a canonical transformation [21. Barros pushed this analysis further by showing that, starting off with this new action, it is possible to solve the arising second class constraint [22]. Then the variables split into two couples $(A, E)$ and $(\chi, \zeta)$. The first couple of canonical variables is a generalisation of the Ashtekar-Barbero connection and the triad. The new variable $\chi$ is the time normal or internal time direction and is given as the normalised space component $\alpha=a$ (in the internal Lorentz indices) of the time component of the tetrad field:

$$
\chi^{a}=-\frac{e^{a 0}}{e^{00}}
$$


and $\zeta$ is its associated canonical momentum. Finally, it is possible to gauge fix $\chi=0$ using (the boost) part of the Lorentz gauge symmetry. This is called the time gauge. And, in that frame, we retrieve exactly the variables and constraints of loop gravity. However, the price of this result is the loss of explicit covariance of the theory.

- Complex Loop Quantum Gravity:

In this approach by Ashtekar, which corresponds to taking $i$ as choice for the Immirzi parameter, we work with a complex connection and complex triad (for a review, see for example [3]). In order to retrieve real results, we must impose reality conditions, which are indeed the second class constraints of the theory. These conditions constrain the measure of the Hilbert space in the quantum theory.

- Covariant Loop Gravity:

In this approach [16, 17, 18, 19], one derives the Dirac bracket to take into account the second class constraints directly in the symplectic structure. This allows to still work with a Lorentz connection. The resulting symplectic structure ignores the space part of the connection/triad and one gets an area spectrum depending only on the boost part of the connection 17]. This result hints toward a $S O(3,1) / S O(3)$ coset structure in the construction of the observables. The split space/boost is done according to the $\chi$ field which becomes a configuration variable that one must take in account when constructing the states of the theory [19]. Despite of these results, the drawback of this approach is that the connection is non-commutative at the classical level. Nevertheless, on one hand, it seems feasible to construct commutative states for the theory [19], and on the other hand, it is possible to find a commutative Lorentz connection, which turns out to be a covariant generalisation of the Ashtekar-Barbero connection [20].

- Spin Foams and the Barrett-Crane model:

The spin foam approach aims toward constructing a space-time model, which would correspond to the space analysis carried out in Loop Quantum Gravity. This would clarify the role of time and the form of the Hamiltonian constraint. The most developped model is up to now the Barrett-Crane model [6, 8] (see [15] for a review). It relies on the (geometric) quantization of a bivector field [6, 6, 13]. From the point of view of the second class constraints, it corresponds to considering them directly at the quantum level [12, 20]. They are then implemented as simplicity constraints on the configuration space of the quantum bivector field. These simplicity constraints amount to restrict the representations of the Lorentz group $S O(3,1)$ in which the bivector can live. They must have $S O(3)$ invariant subspace or, in other words, they must have a realisation in the space $L^{2}(S O(3,1) / S O(3))$ [8]. Such representations are called simple. The resulting boundary states of the theory are $S O(3,1)$ spin networks labelled by simple representations and with simple intertwiners between them [6, 8, 11]. Such functionals are called simple spin networks.

The goal behind the present work is to make a link between these different approaches, more particularly between the spin foam approach and the loop quantum gravity formalism since we would like the spin foam model to help us derive the right dynamics for spin networks. Nevertheless, one is formulated using a Lorentz connection and the other a $S U(2)$ connection. Therefore, one issue would be to understand how get one from the other or, in other words, to understand how the gauge fixing procedure from $S O(3,1)$ to $S O(3)$ works at the quantum level. In the present work, we first introduce projected cylindrical functions of the connection and the $\chi$ field, which depend only on a finite number of arguments taken from those fields, and which can be seen a covariant generalisation of $S U(2)$ spin networks. We show that the boundary states of the Barrett-Crane model, the simple spin networks, can be easily understood in the new context as a particular subset of projected cylindrical functions. From this viewpoint, the study of the Hilbert space of these new functionals can be considered as a first step toward understanding the quantum geometry of spin foams and its link with the canonical formalism. Indeed the Barrett-Crane model is better understood using the time normal and functionals cylindrical in the time normal turn out to be a useful tool in explaining the dynamical and causal structures of the spin foam space-time [14].

Moreover, the same projected cylindrical functions are shown to occur in the covariant loop gravity formalism and we explain how they would help understand this framework and find a suitable hilbert space for its quantum theory. Neverthless, the relation between this covariant approach and the usual $S U(2)$ loop quantum gravity approach will be studied more in details elsewhere 20]. 


\section{Projected Cylindrical Functions}

\subsection{A New Gauge Invariance and its physical meaning}

In the loop quantum gravity approach, we work on a three dimensional space-like hypersurface $\Sigma$-the spaceembedded in a four dimensional space-time $\mathcal{M}$ and we build states of the connection, which will give the geometry of $\Sigma$ at the end of the day. To this purpose, one usually works with cylindrical functions. These are functions, defined on given graphs, which depend on the connection through its holonomies on the edges of the graph. Moreover, one require a gauge invariance of these functions which corresponds to the invariance of the connection state under gauge transformation of the connection. Precisely, given a particular graph with $E$ oriented edges and $V$ vertices and a gauge group $G$, the gauge symmetry reads

$$
\forall k_{i} \in G, \phi\left(U_{1}, U_{2}, \ldots, U_{E}\right)=\phi\left(k_{s(1)} U_{1} k_{t(1)}^{-1}, \ldots, k_{s(E)} U_{E} k_{t(E)}^{-1}\right)
$$

where $s(i)$ and $a(i)$ are respectively the source vertex and the target vertex of the $i$ edge. All a machinery has been developped to study the structure of such functions for compact groups such as $S U(2)$, which is the gauge group of loop quantum gravity. We can introduce a measure -the Haar measure- on the space of such functions (given a particular graph) and we can define the spin networks as a basis of the resulting $L^{2}$ space. Then, we can glue all the different spaces corresponding to all the graphs into a global space of quantum connection state space, which can be viewed as a $L^{2}$ space on a connection space provided with the Ashtekar-Lewandowski measure. However, if we want to develop a covariant formalism, we are bound to use the Lorentz group $S O(3,1)$, which is non-compact, as gauge group. Using directly the Haar measure on such functions gives an infinite result and defining a new invariant measure is not straightforward [1].

In the present work, we follow the covariant approach as explained in the introduction and we would like to use functionals of both the (Lorentz) connection and the time normal $\chi$ to the studied hypersurface. Since it appeared useful to consider cylindrical functions of the connection in the standard approach, we propose in our framework to use cylindrical functions of both the connection and $\chi$. More precisely, given a graph $\Gamma$ with $E$ edges and $V$ vertices, we consider functions depending on the holonomies $U_{i} \in S O(3,1)$ of the connection along the edges and the time normals $x_{i} \in S O(3,1) / S O(3)$ at every node of the graph. Note that $S O(3,1) / S O(3)$ is isomorphic to the upper sheet of the two-sheet hyperboloid in Minkovski space. Considering their transformation under a gauge transformation, a gauge invariant state will be a function with the symmetry:

$$
\forall k_{i} \in S O(3,1), \phi\left(U_{1}, U_{2}, \ldots, U_{E}, x_{1}, \ldots, x_{V}\right)=\phi\left(k_{s(1)} U_{1} k_{t(1)}^{-1}, \ldots, k_{s(E)} U_{E} k_{t(E)}^{-1}, k_{1} \cdot x_{1}, \ldots, k_{V} \cdot x_{V}\right)
$$

Due to the newly introduced $x$ variables, even though the functional $\phi$ is invariant under $S O(3,1)^{V}$, the effective gauge symmetry of $\phi$ is compact. More precisely, it is $\bigotimes_{i=1}^{V} S O(3)_{x_{i}}$. From a physical point of view, the embedding of the hypersurface $\Sigma$ in $\mathcal{M}$ is defined by the whole field $\chi$. But we have forgotten all this information to retain only the value $x_{1}, \ldots, x_{V}$ of $\chi$ at the vertices of the graph so that the embedding of $\Sigma$ is defined at only those points while it is left fuzzy everywhere else. And imposing the normal at a vertex to be $x_{i}$ implies that the symmetry at this vertex is reduced from $S O(3,1)$ to $S O(3)_{x_{i}}$. And it is this effective gauge symmetry that will have to be taken into account when constructing the measure, as we will see in the next paragraph.

A natural choice for the $x$ variables is the vector $x_{0}=(1,0,0,0)$. This corresponds to the time gauge $\chi=0$ and it is the usual gauge fixing that one does when carrying the canonical analysis of the Palatini action through a $3+1$ splitting. In our framework, we don't fix the $x$ variables to a given value but we allow them to vary. This would allow an analysis without gauge fixing and thus explicitely covariant. Moreover, it opens a door to analysing changes of gauge fixing such as the study of a Lorentz boost (see [23] in the case of Loop Gravity) through the shift $x_{1}=\ldots=x_{V}=x_{0}$ to $x_{1}=\ldots=x_{V}=(1, v, 0,0)$.

\subsection{The $L^{2}$ space of Projected Cylindrical Functions}

We would like to give a Hilbert space structure to the space of cylindrical functions defined previously in order to raise them to the status of quantum states of the hypersurface. Indeed, we can use the simple 
Haar measure $d g^{E}$ to integrate functions with the gauge symmetry (雨. And we define the Hilbert space of Projected Cylindrical Functions as the space of $L^{2}$ functions satisfying (4). This induces the scalar product:

$$
\langle\phi \mid \psi\rangle=\int_{S O(3,1)^{E}} \prod_{i} \mathrm{~d} g_{i} \bar{\phi}\left(g_{1}, \ldots, g_{E}, x_{1}, x_{2}, \ldots, x_{V}\right) \psi\left(g_{1}, \ldots, g_{E}, x_{1}, x_{2}, \ldots, x_{V}\right)
$$

One can check that this definition doesn't depend on the choice of $x_{1}, \ldots, x_{V}$. Nevertheless, we have to consider both functions $\phi$ and $\psi$ with the same $x$ arguments i.e to lay in the same embedded hypersurface. Still, this leaves us a freedom in the choice of the $x$ 's and we could fix them all to the unit time vector $x_{0}=(1,0,0,0)$ which is the time gauge.

It is possible to simply use the Haar measure $d g^{E}$ because the effective gauge invariance of functions satisfying (44) is compact, being $S O(3)^{V}$ in the time gauge. What we have done is cancel the non-compact part $S O(3,1) / S O(3)$ of the gauge symmetry. More precisely, it is possible to re-construct the whole function $\phi$ from the function

$$
\tilde{\phi}\left(g_{1}, \ldots, g_{E}\right)=\phi\left(g_{1}, \ldots, g_{E}, x_{0}, x_{0}, \ldots, x_{0}\right)
$$

which satisfies a $S O(3)$ gauge invariance:

$$
\forall k_{i} \in S O(3)_{x_{0}}, \tilde{\phi}\left(g_{1}, g_{2}, \ldots, g_{E}\right)=\tilde{\phi}\left(k_{s(1)} g_{1} k_{t(1)}^{-1}, \ldots, k_{s(E)} g_{E} k_{t(E)}^{-1}\right)
$$

We can visualise this space of functions on a scale of structures:

- There is the space of non-gauge invariant functions, which don't satisfy any symmetry requirement. We can construct a Hilbert space of such functions by simply considering the Haar measure and we get $\mathcal{H}_{\text {non-inv }}=L^{2}\left(d g^{E}\right)$.

- There is the presently introduced $S O(3)$ invariant functions which can be obtained from the previous set of functions by integrating over the $S O(3)$ part of the group elements. We can still use the Haar measure and construct the Hilbert space $\mathcal{H}_{\mathrm{p} r o j}=L_{S O(3) \text { inv }}^{2}\left(d g^{E}\right)$.

- There is the full gauge invariant functions which can be obtained from the previous set by integrating over the remaining $S O(3,1) / S O(3)$ symmetry or equivalently over the $x$ variable. However, we can not use directly the Haar measure and we must construct a quotient measure which make harder to deal with the corresponding Hilbert space [1].

We should note that we can still implement gauge-invariant observables on the space $\mathcal{H}_{\mathrm{proj}}$ in a finite and gauge-invariant way. Indeed, let's consider a gauge invariant function $A\left(g_{1}, \ldots, g_{E}\right)$ invariant under (3). One can consider the matrix elements of $A$ :

$$
\langle\phi|A| \psi\rangle=\int_{S O(3,1)^{E}} \prod_{i} \mathrm{~d} g_{i} \bar{\phi}\left(g_{1}, \ldots, g_{E}, x_{1}, \ldots, x_{V}\right) A\left(g_{1}, \ldots, g_{E}\right) \psi\left(g_{1}, \ldots, g_{E}, x_{1}, \ldots, x_{V}\right)
$$

This doesn't depend on the choice of the $x$ 's and is finite as long as $A$ is bounded. The main issue is then to physically interpret the role of the $x$ variables.

\subsection{Projected Spin Networks}

It is possible to find an orthonormal basis to the introduced $L^{2}$ space. They will have a similar structure as spin network but the symmetry of the intertwiner at a vertex $i$ will be projected from $S O(3,1)$ down to $S O(3)_{x_{i}}$, so that we call the elements of this basis projected spin networks. More precisely, we are going to construct some spin network which will be defined on $S O(3,1)$ but will have $S O(3)$ intertwiners.

Let's choose an oriented graph $\Gamma$ with $E$ edges and $V$ vertices embedded in the space $\Sigma$. Let's construct the holonomies of the connection $A$ along the edges $e_{1}, e_{2}, \ldots, e_{E}$ and denote them $U_{1}, \ldots, U_{E}$. The normal procedure to construct a spin network would be to assign a representation $\mathcal{I}_{i}$ of $S L(2, C)$ to each edge $e_{i}$, then to choose $S L(2, C)$ intertwiners $I_{v}$ for every vertex $v$ of the graph and build the spin network functional by contracting the holonomies $U_{i}$ in the representations $\mathcal{I}_{i}$ with the intertwiners to get a scalar: 


$$
\phi(A)=\bigotimes_{v} I_{v} \bigotimes_{i} D^{\mathcal{I}_{i}}\left(U_{i}\right)
$$

In our case, we further choose a $S U(2)$ representation $j_{i}^{(v)}$ for each edge $e_{i}$ of the graph - for each couple of vertex and incident edge - and a $S U(2)$ intertwiner $i_{v}$ between these representations for each vertex $v$. To glue the holonomies using these intertwiners, we project them at the vertices. To be more precise, let's introduce the representation space $R^{\mathcal{I}}$ of the representation $\mathcal{I}$ of $S L(2, C)$ and the representation space $V^{j}$ of the representation $j$ of $S U(2)$. Unitary irreducible representations of $S L(2, C)$ are infinite dimensional and labelled by a positive integer $n$ and a positive real number $\rho$ (see appendix B for more details). Then, we can decompose $R^{(n, \rho)}$ into $S U(2)$ representations. For this purpose, we choose a particular $S U(2)$ subgroup of $S L(2, C)$ i.e we choose a time normal $x \in S L(2, C) / S U(2)$ and consider the subgroup $S U(2)_{x}$ of elements leaving the vector $x$ invariant (see appendix A for more details). Then:

$$
R^{(n, \rho)}=\bigoplus_{j \geq n} V_{(x)}^{j}
$$

We call $P_{j}^{(x)}$ the orthogonal projector from $R^{(n, \rho)}$ to $V_{(x)}^{j}$. More explicitely, we can express it as

$$
P_{j}^{(x)}=\Delta_{j} \int_{S U(2)_{x}} \mathrm{~d} g \bar{\chi}^{j}(g) D^{(n, \rho)}(g)
$$

where $\Delta_{j}=(2 j+1)$ is the dimension of the $j$ representation, the integration is over the subgroup $S U(2)_{x}$, $D^{(n, \rho)}(g)$ is the matrix of $g$ in the $(n, \rho)$ representation and $\chi^{j}$ is the character of the $j$ representation.

To construct the projected spin network, we insert this projector at both ends of every edges and obtain the following structure around a (3-valent as an example) given vertex $v$ :

$$
\phi\left(U_{1}, U_{2}, U_{3}, \ldots, x_{v}, \ldots\right)=i_{v}^{j_{1} j_{2} j_{3}} \prod_{i=1}^{3}\left|\mathcal{I}_{i} x_{v} j_{i}^{(v)} m_{i}\right\rangle\left\langle\mathcal{I}_{i} x_{v} j_{i}^{(v)} m_{i}\right| D^{\mathcal{I}_{i}}\left(U_{i}\right) \ldots \text { other vertices }
$$

where $|\mathcal{I} x j m\rangle$ is the basis of $V_{(x)}^{j} \hookrightarrow R^{\mathcal{I}}, m$ running from $-j$ to $j$, and where we sum over the $m_{i}$.

It is easy to check that these constructed spin networks satisfy the gauge invariance (4). And a straightforward calculations shows that, once we have chosen an orthonormal set of $S U(2)$ intertwiners, the resulting projected spin networks form an orthonormal basis of the $L^{2}$ space of projected cylindrical functions i.e the scalar product of two spin networks is a delta function on the representations $\mathcal{I}$ and $j$ and on the intertwiners.

Let's emphasize that there is two a priori different $S U(2)$ representations on each edge, at both its ends. When we will tackle the issue of refining the graph, we will then restrict ourself to projected spin networks with a single $S U(2)$ representation on every edge (see section 1.2).

Let's give some examples. The simplest is the one-loop graph with a single vertex. We are looking for functions $\phi$ on $S L(2, C) \otimes S L(2, C) / S U(2)$ satisfying

$$
\forall k \in S L(2, C), \phi(g, x)=\phi\left(k g k^{-1}, k \cdot x\right)
$$

or after having gauge fixed $x$ to, let's say, the time gauge, we can write the gauge invariance on the gauge fixed function $\tilde{\phi}(g)=\phi\left(g, x_{0}\right)$ :

$$
\forall h \in S U(2), \tilde{\phi}(g)=\tilde{\phi}\left(h g h^{-1}\right)
$$

The projected spin network construction gives functions labelled by one $S L(2, C)$ representation $\mathcal{I}=(n, \rho)$ and one $S U(2)$ representation $j$. Instead of considering the character of the representation $(n, \rho)$, we restrict ourself to the trace over the subspace $V_{x}^{j}$ :

$$
\phi_{j}^{(n, \rho)}(g, x)=\operatorname{Tr}_{V_{x}^{j}}^{(n, \rho)}(g)
$$

Another example is the $\Theta$ graph. It has two 3-valent vertices $A$ and $B$. We choose three $S L(2, C)$ representations and three $S U(2)$ representations. The resulting projected spin networks is given by taking the matrix elements of the three $S L(2, C)$ group elements in the $S L(2, C)$ representation restricted to the spaces $V_{x}^{j}$ and 
contract them with the two Clebsh-Gordon coefficients which are the unique three-valent $S U(2)$-intertwiner (up to normalisation).

\subsection{Going down to $S U(2)$ spin networks}

One interesting issue is how these newly defined projected spin networks could relate to the usual $S U(2)$ spin networks which are a basis of the states of quantum geometry in the usual formulation of loop quantum gravity.

In fact, the projected spin networks easily go down to $S U(2)$ spin network. More precisely, in the special gauge where all $x$ variables are the same, let's say equal to $x_{0}$, it is possible to restrict the group variables to live in the $S U(2)_{x_{0}}$ subgroup and consider the (projected) functionals as functions over $S U(2)^{E}$. Due to the gauge invariance (画, they are effectively cylindrical functions on $S U(2)$. Even better, projected spin networks with edges labelled by representations $\left(\mathcal{I}_{e}=\left(n_{e}, \rho_{e}\right), j_{e}\right)$ (the two $S U(2)$ representations at the two ends of one given edge must match, else the resulting $S U(2)$ spin network is 0 , so we choose only one $S U(2)$ representation per edge) simply reduces to a $S U(2)$ spin network with edges labelled by the $S U(2)$ representations $j_{e}$, the vertices still labelled by the same $S U(2)$ intertwiners. Moreover, this procedure is obviously independent from the choice of gauge as long as all the $x$ 's are chosen to be equal: one gets the exact same $S U(2)$ spin network.

Interestingly, the $S L(2, C)$ representations $\left(n_{e}, \rho_{e}\right)$ coming into the definition of the initial projected spin network don't intervene in the definition of the resulting $S U(2)$ spin network. Choosing other representations $\left(\tilde{n}_{e}, \tilde{\rho}_{e}\right)$ still gives the same $S U(2)$ spin network (as long as we have chosenthe same $j_{e}$ ). Thus, they don't seem to describe the $3 \mathrm{~d}$ geometry but more how it changes under Lorentz boost: they can be understood as the space-time embedding of the studied space slice (or the structure of the space-time around that slice). This is a reason why the present formalism leads to a covariant definition of the $S U(2)$ spin networks.

\subsection{Theory for arbitrary group}

We start by recalling some group theory to introduce the maximal compact subgroup of a group $G$ and its basic properties. The following elements are taken from [25]. We consider a linear connected reductive group $G$ i.e a closed connected group of real or complex matrices that is stable under conjugate transpose. We note $\mathcal{G}$ its Lie algebra. The inverse conjugate transpose, denoted $\Theta$, is an automorphism of $G$ and is called the Cartan involution. We note that $\Theta^{2}=1$. Let

$$
K=\{g \in G \mid \Theta g=g\} .
$$

$K$ is a compact connected and is a maximal compact subgroup of $G$. For example, it is $S O(3)$ in the case of $G=S O(3,1)$ and $S U(2)$ in the case of $G=S L(2, \mathbf{C})$. The differential $\theta$ of $\Theta$ at the identity 1 is an automorphism of $\mathcal{G}$, given as the negative conjugate transpose. Since $\theta^{2}=1$, we have the Cartan decomposition for $\mathcal{G}$

$$
\mathcal{G}=p \oplus m,
$$

where $p$ and $m$ are the eigenspaces corresponding to the eigenvalues 1 and -1 of $\theta . p$ consists of the skewHermitian members of $\mathcal{G}, m$ of the Hermitian elements, and it is easy to derive the following relations:

$$
\begin{array}{ccc}
{[p, p]} & \subset & p \\
{[p, m]} & \subset & m \\
{[m, m]} & \subset & p
\end{array}
$$

The Cartan decomposition for $G$ is the map

$$
\begin{array}{ccc}
K \times m & \rightarrow & G \\
(k, u) & \rightarrow & k \exp (u)
\end{array}
$$

It is a diffeomorphism onto $G$, which identifies the quotient $X=G / K$ of the group $G$ under the left action of $K$ to the space $m$. 
A projected cylindrical function will be defined on a graph $\Gamma$ - with $E$ oriented edges and $V$ vertices - and will depend on a group element for each edge and a spin variable $x \in G / K$ for each vertex and will have the gauge symmetry:

$$
\forall k_{i} \in G, \phi\left(g_{1}, g_{2}, \ldots, g_{E}, x_{1}, \ldots, x_{V}\right)=\phi\left(k_{s(1)} g_{1} k_{t(1)}^{-1}, \ldots, k_{s(E)} g_{E} k_{t(E)}^{-1}, k_{1} \cdot x_{1}, \ldots, k_{V} \cdot x_{V}\right)
$$

Such a function can be constructed from an arbitrary function over $G^{\otimes E}$ by (left-)integrating over the $K$ part of the $E$ group elements. The gauge fixing corresponds to fixing all the $x$ variables to some arbitrary values and the time gauge amounts to fix all $x$ to the equivalence class [Id] of the identity i.e the group $K$ itself. The measure we introduce is the Haar measure $d g^{\otimes E}$. The resulting Hilbert space is the space of $L^{2}$ functions satisfying the required symmetry. And we see that we have managed to sidestep the problem of dealing with a non-compact gauge group.

We now would like to also generalise the construction of the projected spin networks. To this purpose, let's see how a representation of $G$ decomposes over representations of $K$. To be more precise, let $G$ be a linear connected reductive group and $\pi$ a representation of $G$ on a Hilbert space $V$. When $K$ acts by unitary operators, then we can decompose $\pi$ into irreducible representations of $K$ [25] (chapter 8):

$$
\pi=\sum_{\tau \in \hat{K}} n_{\tau} \tau
$$

where $\hat{K}$ is the set of equivalence class of irreducible representations of $K$ and $n_{\tau} \in \mathbf{N} \cup\{+\infty\}$ is the multiplicity of each representation. The equivalence classes $\tau$ that have a positive multiplicity are called $K$ types. Moreover, if $\pi$ is an irreducible unitary representation, all the multiplicities $n_{\tau}$ are finite and satisfy $n_{\tau} \leq \operatorname{dim} \tau$.

We can further introduce $K$-finite vectors which are vectors $v$ such that $\pi(K) v$ spans a finite-dimensional space. Then, for unitary representations (or more generally so-called admissible representations), all $K$ finite vectors are $C^{\infty}$ vectors, the space of $K$-finite vectors is stable under $\pi(\mathcal{G})$ and every matrix coefficient $g \rightarrow(\pi(g) u, v)$, with $u K$-finite, is a real analytic function on $G$.

We are then ready to introduce the projected spin networks. We choose $E$ group elements $g_{1}, \ldots, g_{E}$ on the edges of the graph, and $V$ elements $x_{1}, \ldots, x_{V}$ of the coset $G / K$. We further choose $E$ representations $\mathcal{I}$ of $G$, and $2 E$ representations of $K$. We can follow the exact same construction as in the $S L(2, C)$ case, choosing $V K$-intertwiners and contracting them using the matrix element of $\pi^{\mathcal{I}_{i}}\left(g_{i}\right)$ in a basis of the representation space of the $K$-subgroups corresponding to the choice of $x$ 's. Finally, due to the $K$-finiteness of the vectors used in the construction, the resulting function is perfectly well-defined.

\section{The Barrett-Crane model and its boundary states}

\subsection{Simple Spin Networks}

The Barrett-Crane model is a spin foam model. More precisely, it is a theory of space-time based on a simplicial discretisation with representations of $S L(2, C)$ living on the faces (triangles) of the decomposition (see [15] for a review). Its boundary states are 4 -valent simple spin networks i.e $S L(2, C)$ spin networks with simple representations and simple intertwiners. The link of the model with general relativity is that it appears as a discretisation of a constrained BF theory equivalent to the usual Palatini action $[5,13$.

There exists two Lorentzian models. The first one [9] is based on simple representation that can be represented on the space of $L^{2}$ functions over the 2-sheet hyperboloid $S L(2, C) / S U(2)$. The induced representations are of the type $(0, \rho)$ (see appendix B for a review of the irreducible unitary representations of $S L(2, C)$ ). The second one [10] uses the space of $L^{2}$ functions over the one-sheet hyperboloid $S L(2, C) / S U(1,1)$ and induces all the simple representations $(n, 0)$ and $(0, \rho)$. They can both be expressed in term of a field theory over $S L(2, C)$. In the present work, we will deal only the first model because the structure of its boundary term allows a simple interpretation in terms of space-like hypersurface, as we will see later.

We are now going to have a look at the simple spin networks that arise in this Lorentzian model and explain how they enter in the framework of projected spin networks. Here, we will only review the elements necessary to the present analysis. [1] gives a general presentation of simple spin networks and one can look 
in [8, 11] for a precise account of Lorentzian simple spin networks. The results are as follows. Simple spin networks are defined by simple representations $\rho_{e}(S L(2, C)$ irreducible representations containing a $S U(2)$ invariant subspace) living on the edges of the graph. At the vertices, we contract the matrix elements of the group elements using simple intertwiners. The interesting thing is that these intertwiners can be written as an intregral over $S L(2, C) / S U(2)$. More precisely, to compute the simple spin network, one chooses a $x_{v} \in S L(2, C) / S U(2)$ for each vertex. Then, for each edge $e$, one computes the corresponding Kernel (as defined in the appendix (D)

$$
K_{\rho_{e}}\left(x_{s(e)}, g_{e} x_{t(e)}\right)=\left\langle\rho_{e}(j=m=0)\left|\left(x_{s(e)}\right)^{-1} g_{e} x_{t(e)}\right| \rho_{e}(j=m=0)\right\rangle
$$

Finally one considers the product of these Kernels and integrate over the $x$ variables. It appears that if one forgets about the final integration, one has exactly computed the projected spin networks defined earlier in the special case when one sets the $S U(2)$ representation to the trivial one $j=0$ everywhere. In particular, one can interpret the $x$ variables as the time direction and therefore the simple spin networks as describing a space-like hypersurface. If one had considered the simple spin networks for $S L(2, C) / S U(1,1)$, one would have been correspondingly describing a time-like hypersurface.

As projected spin networks, the simple spin networks satisfy the symmetry (仼. Nevertheless, they have an extra symmetry: at each vertex, they are invariant under $S U(2)^{n}$ instead of a simple $S U(2), n$ being the number of edges linked to that given vertex. In the spin foam framework, this comes from the simplicity constraints i.e corresponds to the implementation of the second-class constraints.

One can easily check the orthonormality of the simple spin networks for the scalar product (8) using the formula of appendix C. Indeed, when computing the scalar product of two simple spin networks, once we have fixed the $x$ variables at all the vertices, we can split the integral over $S L(2, C)^{E}$ into the product of $E$ integrals over $S L(2, C)$ of the product of the two Kernels corresponding to the same edge but to the two different spin networks, then

$$
\int_{S L(2, C)} d g K_{\rho}\left(x_{d(e)}, g x_{t(e)}\right) K_{\rho^{\prime}}\left(x_{d(e)}, g x_{t(e)}\right)=\frac{\delta\left(\rho-\rho^{\prime}\right)}{\rho^{2}} K_{\rho}\left(x_{d(e)}, x_{d(e)}\right)=\frac{\delta\left(\rho-\rho^{\prime}\right)}{\rho^{2}}
$$

which shows the orthonormality of the simple spin networks. A simple cylindrical function is obtained by integrating over the representations $\rho$ the defined simple spin network with $L^{2}\left(\rho^{2} d \rho\right)$ distributions using the Plancherel measure $\rho^{2} d \rho$. As an example, let's consider a single edge and the corresponding simple spin network $K_{\rho}(x, g y)$. A $L^{2}$ function $f$ is defined by a $\alpha \in L^{2}\left(\rho^{2} d \rho\right)$ as

$$
f(g, x, y)=\int \rho^{2} d \rho \alpha(\rho) K_{\rho}(x, g y)
$$

and its $L^{2}$ norm is the $L^{2}$ norm of the function $\alpha$.

Let's also point out that the function given by a single Kernel $f(g, x, y)=K(x, g y)$ is a projected cylindrical function: it is possible to consider open spin networks (which are still gauge invariant) within the context of projected cylindrical spin networks.

\subsection{3d geometry from the spin foam boundary?}

An interesting question in order to understand the physical content of these simple spin networks is what kind of "quantum" 3d geometry do they describe. Indeed, they are supposed to be the quantum state of the hypersurface. So what data do they contain?

First, one can look at the $S U(2)$ spin networks induced by the simple spin networks following the same procedure as in the previous section: let's choose a gauge where all the $x$ 's are fixed to $x_{0}$ and study the functions restricted to $S U(2)_{x_{0}}$ group elements. Then, one finds $S U(2)$ spin networks with edges labelled by the trivial representation $j=0$ : the simple spin networks don't seem to carry any information on the $S U(2)$ restriction of the connection. Indeed, the simple spin networks can be considered as the Fourier decomposition of the requirement that the $S L(2, C)$ group element live in $S U(2)$. More precisely, one has the equation:

$$
\delta_{\mathcal{H}_{+}}\left(x_{d(e)}, g_{e} x_{a(e)}\right)=\frac{1}{2 \pi^{2}} \int_{0}^{\infty} \rho^{2} d \rho K_{\rho}\left(x_{d(e)}, x_{a(e)}\right)
$$


which says that the $K_{\rho}\left(x_{d(e)}, x_{a(e)}\right)$ (one edge of a simple spin network) can be seen as the Fourier transform of the requirement that $g_{e}$ must define a parallel transport, along the edge $e$, which takes $x_{d(e)}$ to $x_{a(e)}$. However, there exists a whole $S U(2)$ subgroup of such group elements among which $g_{e}$ can be randomly chosen.

Due to this, simple spin networks don't give amplitudes differentiating between the $S U(2)$ part of the group elements: they are not similar to using $S U(2)$ spin networks, which are interpreted as giving an amplitude on the $S U(2)$ connection defining the $3 \mathrm{~d}$ geometry. On the other hand, they describe how the space hypersurface changes under Lorentz boosts i.e under change of embedding into the space-time. They seem more like an orthogonal information: $S U(2)$ spin network seems an extra information one would have to impose by hand. $S U(2)$ spin networks don't seem to arise naturally in the Barrett-Crane model and that's why it seems more natural to compare the spin foam setting with the covariant canonical analysis of the Palatini action as done by Alexandrov [16, 17, 18, 19.

Still, thinking the Euclidean Barrett-Crane model based on the group $\operatorname{Spin}(4)=S U(2)_{L} \otimes S U(2)_{R}$, simple representations (which contain a vector invariant under the diagonal $S U(2)$ subgroup) are representations $(j, j)$ (same representations for $S U(2)_{L}$ and $\left.S U(2)_{R}\right)$ and simple spin networks labelled by $\left(j_{e}, j_{e}\right.$ ) are thought to go down to $S U(2)$ spin networks labelled by the representations $j_{e}$ (because the areas defined by the Casimirs is the same for such a choice): we don't restrict to the diagonal $S U(2)$ which is the space symmetry group but to $S U(2)_{L}$ (or $S U(2)_{R}$ which would be equivalent). Therefore, maybe a $S U(2)$ spin network interpretation of Lorentzian spin networks is still possible considering another type of restriction of the group elements. A more careful study of the gauge fixing procedure would be needed for such purpose.

\section{Towards Covariant Loop Quantum Gravity?}

\subsection{Fock Space of Projected Cylindrical Functions}

At the end of the day, one would like to have a Hilbert space of quantum states of geometry. And more precisely, in loop-like approaches, we are looking for a Hilbert space of quantum states of the connection, which should be obtained by summing over all the spin networks states i.e summing the Hilbert spaces corresponding to all the different graph. In Loop Quantum Gravity, through the Ashtekar-Lewandowski construction, it is possible to make this space appear as a $L^{2}$ space [2]. This even works for any compact group. But this approach fails in the case of non-compact groups [1].

In the case of projected cylindrical functions, it is nevertheless possible to give a Fock space structure to the sum over graphs. This is due to the use of the normal $S L(2, C)$ Haar measure (and not a quotient measure as in [1]) allowed by the compact effective gauge invariance. More precisely, let's choose a graph $\Gamma$ and a subgraph $\Gamma_{1}$, a $L^{2}$ cylindrical function $f$ on $\Gamma$ and $L^{2}$ cylindrical function $\varphi$ on $\Gamma_{1}$. The action of the annihilation operator $a_{\varphi}$ on the function $f$ will give a $L^{2}$ cylindrical function on $\Gamma^{\prime}=\Gamma \backslash \Gamma_{1}$ defined as the set of edges of $\Gamma$ which are not in $\Gamma_{1}$ linked by the needed vertices of $\Gamma$ (vertices which are not in the interior of $\Gamma_{1}$ :

$$
a_{\varphi} f\left(g_{f \in \Gamma^{\prime}}, x_{w \in \Gamma^{\prime}}\right)=\int \prod_{e \in \Gamma_{1}} \mathrm{~d} g_{e} \bar{\varphi}\left(g_{e \in \Gamma_{1}}, x_{v \in \Gamma_{1}}\right) f\left(g_{e \in \Gamma_{1}}, g_{f \notin \Gamma_{1}}, x_{v \in \Gamma}\right)
$$

where the $x_{v \in \Gamma_{1}}$ variables are taken to be the same for $\varphi$ and $f$.

We can also write creation operators acting on a given graph $\Gamma$, adding edges to it by acting on $L_{S O(3) \text { inv }}^{2}(\Gamma)$ with a cylindrical function depending only on the added edges and the corresponding vertices. This action amounts to multiply the two functions together. One must be careful that we are not gluing two graphs together along some common edges, but only adding some edges: the only thing in common are vertices. This other situation would be more complicated and would need some convolution product, which will not be investigated here.

Finally, we have seen it is possible to endow the sum over graphs of all $L^{2}$ projected cylindrical functions of a Fock space structure. 


\subsection{Refinement and the bivalent vertex problem}

In this paragraph, we will tackle the issue of refining the projected cylindrical functions. More precisely, I mean that given a graph, a projected cylindrical function is projected only at the vertices of the graph and we will see how to project it at virtually every point of the graph precising the time direction at all points of the graph. This would correspond to (fully) projected spin networks as introduced in 19] for a proposal of Hilbert space for covariant loop gravity.

We will therefore study bivalent vertices and how to pass between the space of functions depending on one edge and its two vertices and the ones depending on two edges linked with a bivalent vertex (while ignoring other edges of the graph):

$$
\begin{array}{cccc}
f(g, x, y) & = & & f\left(k g h^{-1}, k x, h y\right) \\
& \uparrow & ? & \\
\phi\left(g_{1}, g_{2}, x, y, z\right) & = & \phi\left(a g_{1} c^{-1}, c g_{2} b^{-1}, a x, b y, c z\right)
\end{array}
$$

The most natural operation between the two sets is to start with a function $f$ and define a $\phi$ function by contracting its two group elements:

$$
f \rightarrow \phi\left(g_{1}, g_{2}, x, y, z\right)=f\left(g=g_{1} g_{2}, x, y\right)
$$

One can write an "inverse" to this operation to go from a $\phi$ function to a $f$ function by integrating over $z$. More precisely, $\int d z \phi\left(g_{1}, g_{2}, x, y, z\right)$ depends on $g_{1}$ and $g_{2}$ through only $g=g_{1} g_{2}$. Moreover, we can express this same integral using a kind of convolution:

$$
\phi \rightarrow f(g, x, y)=\int_{S L(2, C)} \mathrm{d} \tilde{g} \phi\left(\tilde{g}, \tilde{g}^{-1} g, x, y, z\right)=\int_{\mathcal{H}_{+}} \mathrm{d} z \phi\left(g_{1}, g_{2}, x, y, z\right)
$$

However, this is the contrary of what we are looking for since we would like to tell what is the value of the intermediate variable $z$ i.e insert a dependence over $z$ instead of ignoring it as in (28). We are going to see how this can be done working on projected spin networks. To start with, let's focus on a given edge of a projected spin network. Three variables live on this edge: a group element $g$ and two time normals $x$ and $y$ at its two vertices. The spin network further depends on a $S L(2, C)$ representation $\mathcal{I}$ and two $S U(2)$ representations $j_{1}, j_{2}$ so that the function reads

$$
f(g, x, y, \ldots)=\left\langle\mathcal{I} x j_{1} m_{1}\left|D^{\mathcal{I}}(g)\right| \mathcal{I} y j_{2} m_{2}\right\rangle \ldots
$$

We are going to create a bivalent vertex in the middle of this edge by inserting the Identity

$$
\forall j, \operatorname{Id}_{R^{\mathcal{I}}}=\frac{1}{\Delta_{j}} \int_{\mathcal{H}_{+}} \mathrm{d} z P_{(z)}^{j}
$$

This will work iff $j_{1}=j_{2}=j$. Indeed, inserting a bivalent vertex means also imposing a $S U(2)$ invariance at this vertex. And this imposes to have the same $S U(2)$ representation on both side. Therefore, we are considering projected spin network with a unique $S U(2)$ representation for each edge. Then, we can consider the following function

$$
\phi\left(g_{1}, g_{2}, x, y, z, \ldots\right)=\frac{1}{\Delta_{j}}\left\langle\mathcal{I} x j_{1} m_{1}\left|D^{\mathcal{I}}\left(g_{1}\right) P_{(z)}^{j} D^{\mathcal{I}}\left(g_{2}\right)\right| \mathcal{I} y j_{2} m_{2}\right\rangle \ldots
$$

Once we use the operation (29) on this function, we fall back onto our feet and find back the function $f(g, x, y)$ we started with. This way, we can precise the normal $z$ at a point on an edge of the graph. We would like to repeat this process infinitely many times to fully project our cylindrical function. Nevertheless, a problem is that this "projection" operation is not straightforwardly consistent with the scalar product i.e it doesn't respect (commute with) the $L^{2}$ structure we are using. For the sake of the simplicity of the notations, we will look only at simple spin networks, but the following is also valid for any projected spin network.

Let's consider a 2-edge graph with an edge going from $x$ to $z$ labelled with a $L^{2}\left(\rho^{2} d \rho\right)$ representation distribution $\alpha(\rho)$ and an edge going from $z$ to $y$ labelled with $\beta(\rho)$ : 


$$
\phi\left(g_{1}, g_{2}, x, y, z\right)=\int \rho_{1}^{2} d \rho_{1} \int \rho_{2}^{2} d \rho_{2} \alpha\left(\rho_{1}\right) \beta\left(\rho_{2}\right) K_{\rho_{1}}\left(x, g_{1} z\right) K_{\rho_{2}}\left(z, g_{2} y\right)
$$

$\phi$ is a $L^{2}$ function using the measure defined for the 2-edge graph and its norm is

$$
|\phi|^{2}=\left(\int \rho^{2} d \rho \alpha^{2}(\rho)\right)\left(\int \rho^{2} d \rho \beta^{2}(\rho)\right)
$$

We coarse grain it using (29) and we obtain the function $f$ defined on a 1-edge graph going from $x$ to $y$ :

$$
f(g, x, y)=\int \rho^{2} d \rho \alpha(\rho) \beta(\rho) K_{\rho}(x, g y)
$$

The norm of $f$ using the measure defined for the 1-edge graph is

$$
|f|^{2}=\int \rho^{2} d \rho \alpha^{2}(\rho) \beta^{2}(\rho)
$$

which is not finite in general i.e $f$ is not automatically a $L^{2}$ function! Nevertheless, $f$ is $L^{2}$ if $\alpha$ and $\beta$ are both in $L^{4}\left(\rho^{2} d \rho\right)$. And if we want to refine more and more, we will have to choose functions in $L^{2} \cap L^{4} \cap L^{6} \cap \ldots$, as for example functions with compact support. It will still be possible to refine projected cylindrical functions using the procedure described for projected spin networks. But the $L^{2}$ norm of the refined functions will not be equal.

There is another point of view, which amounts to impose that the norm is conserved by refining. Indeed the coarse graining projection (29) is obviously generalised to graphs more and more refined (with more and more bivalent vertices) and allow to define projections $p_{\Gamma_{1} \Gamma_{2}}$ with $\Gamma_{2} \subset \Gamma_{1}\left(\Gamma_{2}\right.$ is a coarse grained version of $\Gamma_{1}$ : we have integrated out some bivalent vertices) which take $L_{\Gamma_{1}}^{1}$ functions to $L_{\Gamma_{2}}^{1}$ functions. Thus, one can define sequences of consistent $L^{1}$ functions (which project on one another) whose integral (using the Haar measure) is conserved under refining of the graph. This means we can define a (generalised) measure $d \mu_{\infty}$ over such consistent sequences by saying that the integral of a sequence is simply the integral of one of its functions (since these integrals are all the same). We can also define a Hilbert space $L^{2}\left(d \mu_{\infty}\right)$. The drawback of this approach is that the refining of such $L^{2}$ functions is not (29) anymore but

$$
\phi \rightarrow f=\left(\int d \tilde{g} \phi^{2}\right)^{\frac{1}{2}}
$$

which doesn't lead to the "nice" (easily interpreted) refining of the projected spin network described above.

\subsection{Projected Spin Networks as Area Eigenvectors}

An argument for the use of projected cylindrical functions in covariant loop gravity is their link with Alexandrov's covariant approach to canonical loop gravity [16]. More precisely, in his approach, one deals with a $S L(2, C)$ connection $A_{i}^{X}$ ( $i$ being the space index and $X$ a $s l(2, c)$ index) and a tetrad field $P_{Y}^{j}$ and one can derive a Dirac bracket, taking into account the second class constraint, which projects onto the boost part of the connection:

$$
\left\{A_{i}^{X}(x), P_{Y}^{j}(y)\right\}_{D}=I_{(\chi) Y}^{X} \delta_{i}^{j} \delta(x, y)
$$

where $\chi$ is the time normal field and the projector $I_{(\chi) Y}^{X}$ is 1 when $X$ and $Y$ are in the boost part orthogonal to $S U(2)_{\chi}$ and 0 in all other cases (see [17] for more details).

Here the projected cylindrical functions allow a discrete representation of this commutation relation. More precisely, let's choose a finite number of points on the manifold. Then, by considering graphs whose set of vertices contains those points, one gets a representation of the commutation relations at the chosen points. Moreover, one can define the area operator at the vertices of the graph. Indeed for each couple of vertex and incident edge, the projected spin networks are eigenvectors of the area operator of a surface intersecting the edge at the vertex and one gets the area spectrum derived in [17]: 


$$
\text { Area } \sim \sqrt{j(j+1)-n^{2}+\rho^{2}+1}
$$

This choice of Hilbert space is different from the approach of Alexandrov in [19] who tries to build a representation for the commutation relation at all points of space. This would correspond to an infinite refinement limit of the present projected cylindrical function as described in the previous paragraph. The advantage of the present approach is that the constructed functionals are truly cylindrical in that they depend on both the connection and time normal field by a finite number of variables.

\section{Conclusions \& Outlooks}

Projected cylindrical functions, as presented here, are a first step toward constructing a suitable Hilbert space for covariant loop gravity. They are properly defined in the sense that they depend on the connection and the time normal field by a finite number of arguments. They form a Hilbert space whose basis, the projected spin networks, can be considered as a covariant generalisation of $S U(2)$ spin networks. We also studied an infinite refinement limit of those spin networks in which one would know the value of the time normal on the whole manifold instead of knowing it at a finite number of points. It was shown that either this limit doesn't allow an interpretation as a $L^{2}$ space or the refining is not the obvious one.

Then, it would be interesting to fully study the introduced functionals in the framework of Alexandrov's framework and, to start with, derive the algebra of those projected cylindrical functions (their Dirac bracket). Moreover the use of the time normal field $\chi$ allowing a straightforward geometrical interpretation of the states shows a clear link with the spin foam formalism and reinforce the hope of an explicit correspondence between the structures of loop gravity and the ones arising from the Barrett-Crane model, even though it isn't not straightforward to derive $S U(2)$ spin networks from the spin foam boundary states.

It would also be interesting to apply history phase space techniques to covariant canonical loop gravity since it takes in account explicitely the embedding (here given by the $\chi$ field) of the studied hypersurface. This could yield the searched link with the spin foam space-time structures.

Finally, using an explicitely covariant formalism opens the door to the systematic study of space-time related issues such as transformations of areas under Lorentz boosts, as studied in Loop Quantum Gravity [23.

\section{Acknowledgments}

The author is much grateful to Daniele Oriti and Carlo Rovelli for many stimulating discussions and comments on the manuscript.

\section{A $S O(3,1) / S O(3)$}

For $x$ on the future hyperboloid $\mathcal{H}^{+}$i.e a time-like future-oriented unit 4-vector, we can define its stabiliser under the action $g . x$ of $S O(3,1)$ :

$$
S O(3)_{x}=\{h \in S O(3,1) / h \cdot x=x\}
$$

The transformation law of $S O(3)_{x}$ under the action of $S O(3,1)$ is

$$
\forall g \in S O(3,1), S O(3)_{g . x}=g S O(3)_{x} g^{-1}
$$

Then $\mathcal{H}^{+}$can be seen as the quotient $S O(3,1) / S O(3)$ of $S O(3,1)$ by the left action of the subgroup $S O(3)$ - the canonical $S O(3)$ subgroup i.e the stabiliser of $x_{0}=(1,0,0,0)$. Then for $\left[g_{0}\right] \in S O(3,1) / S O(3)$ and $g \in S O(3,1)$, we have:

$$
S O(3)_{\left[g_{0}\right]}=\left\{k \in S O(3,1) / k \cdot\left[g_{0}\right]=\left[g_{0}\right]\right\}=\left\{k \in S O(3,1) / S O(3) g_{0} k=S O(3) g_{0}\right\}
$$




$$
S O(3)_{g \cdot\left[g_{0}\right]}=S O(3)_{\left[g_{0} g^{-1}\right]}=g S O(3)_{\left[g_{0}\right]} g^{-1}
$$

Now, if one considers an irreducible unitary representation $R^{\mathcal{I}}$ of $S O(3,1)$, we can decompose it on the irreducible (finite-dimensional) representations of $S O(3)_{x}$ for a given vector $x$ :

$$
R^{\mathcal{I}}=\bigoplus_{j} V_{(x)}^{j}
$$

So one can choose a nice basis of $R^{\mathcal{I}}$ by considering the canonical basis of the spaces $V_{(x)}^{j}$. This way, one gets a basis $|\mathcal{I} x j m\rangle$. And using (41), we can related these basis for different choices of $x$ by

$$
|\mathcal{I}(g . x) j m\rangle=D^{\mathcal{I}}(g)|\mathcal{I} x j m\rangle
$$

\section{B Irreducible representations of the Lorentz group}

From the initial generators of the $s o(3,1)$ algebra $T_{X}=\left(A_{a},-B_{a}\right)$, we can introduce the following generators :

$$
\begin{aligned}
& H_{+}=i B_{1}-B_{2}, \quad H_{-}=i B_{1}+B_{2}, \quad H_{3}=i B_{3}, \\
& F_{+}=i A_{1}-A_{2}, \quad F_{-}=i A_{1}+A_{2}, \quad F_{3}=i A_{3} .
\end{aligned}
$$

These new generators commute in the following way:

$$
\begin{aligned}
& {\left[H_{+}, H_{3}\right]=-H_{+}, \quad\left[H_{-}, H_{3}\right]=H_{-}, \quad\left[H_{+}, H_{-}\right]=2 H_{3},} \\
& {\left[H_{+}, F_{+}\right]=\left[H_{-}, F_{-}\right]=\left[H_{3}, F_{3}\right]=0 \text {, }} \\
& {\left[H_{+}, F_{3}\right]=-F_{+}, \quad\left[H_{-}, F_{3}\right]=F_{-} \text {, }} \\
& {\left[H_{+}, F_{-}\right]=-\left[H_{-}, F_{+}\right]=2 F_{3},} \\
& {\left[F_{+}, H_{3}\right]=-F_{+}, \quad\left[F_{-}, H_{3}\right]=F_{-},} \\
& {\left[F_{+}, F_{3}\right]=H_{+}, \quad\left[F_{-}, F_{3}\right]=-H_{-}, \quad\left[F_{+}, F_{-}\right]=-2 H_{3} \text {. }}
\end{aligned}
$$

The irreducible infinite dimensional representation of the Lorentz group are characterized by two numbers $\left(l_{0}, l_{1}\right)$, where $l_{0} \in \mathbf{N} / 2$ and $l_{1} \in \mathbf{C}$. In the space $\mathcal{H}_{l_{0}, l_{1}}$ of this representation one can introduce an orthonormal basis

$$
\{|j, m\rangle\}, \quad m=-j,-j+1, \ldots, j-1, j, \quad j=l_{0}, l_{0}+1, \ldots
$$

such that the generators introduced above act in the following way [27]:

$$
\begin{aligned}
H_{3}|j, m\rangle & =m|j, m\rangle, \\
H_{+}|j, m\rangle & =\sqrt{(j+m+1)(j-m)}|j, m+1\rangle, \\
H_{-}|j, m\rangle & =\sqrt{(j+m)(j-m+1)}|j, m-1\rangle, \\
F_{3}|j, m\rangle & =\gamma_{(j)} \sqrt{j^{2}-m^{2}}|j-1, m\rangle+\beta_{(j)} m|j, m\rangle-\gamma_{(j+1)} \sqrt{(j+1)^{2}-m^{2}}|j+1, m\rangle, \\
F_{+}|j, m\rangle & =\gamma_{(j)} \sqrt{(j-m)(j-m-1)}|j-1, m+1\rangle+\beta_{(j)} \sqrt{(j-m)(j+m+1)}|j, m+1\rangle \\
& +\gamma_{(j+1)} \sqrt{(j+m+1)(j+m+2)}|j+1, m+1\rangle, \\
F_{-}|j, m\rangle & =-\gamma_{(j)} \sqrt{(j+m)(j+m-1)}|j-1, m-1\rangle+\beta_{(j)} \sqrt{(j+m)(j-m+1)}|j, m-1\rangle \\
& -\gamma_{(j+1)} \sqrt{(j-m+1)(j-m+2)}|j+1, m-1\rangle,
\end{aligned}
$$

where

$$
\beta_{(j)}=-\frac{i l_{0} l_{1}}{j(j+1)}, \quad \gamma_{(j)}=\frac{i}{j} \sqrt{\frac{\left(j^{2}-l_{0}^{2}\right)\left(j^{2}-l_{1}^{2}\right)}{4 j^{2}-1}} .
$$


The unitary representations correspond to two cases:

$$
\begin{array}{ccc}
\text { 1) } & (n, i \rho), \quad n \in \mathbf{N} / 2, \rho \in \mathbf{R} & \text { - principal series, } \\
2) & (0, \rho), \quad|\rho|<1, \rho \in \mathbf{R} & \text { - supplementary series. }
\end{array}
$$

The principal series are called as such for they are the ones involved in the Plancherel decomposition of $L^{2}$ functions over $S L(2, C)$ [26]:

$$
f(g)=\frac{1}{8 \pi^{4}} \sum_{n} \int \operatorname{Tr}\left[F(n, \rho) D^{n, \rho}\left(g^{-1}\right)\right]\left(n^{2}+\rho^{2}\right) d \rho
$$

where $\left(n^{2}+\rho^{2}\right) d \rho$ is the Plancherel measure over the principal series of representations and the Fourier transform $F$ is defined as

$$
F(n, \rho)=\int f(g) D^{n, \rho}(g) d g
$$

Simple representations are the principal unitary representations with the Casimir $-i l_{0} l_{1}=n \rho=0$. These are the representations coming into the spin foam models as explained in [8]. There are obvisouly two series of such representations: the $(n, 0)$ discrete series and $(0, \rho)$ continuous series. They both share the fact that all their coefficients $\beta_{(j)}$ are 0 .

\section{The $S L(2, C)$ Kernel}

Simple representations of the $(0, \rho)$ type have a unique $S U(2)$ invariant vector, noted $|\rho j=0\rangle$, and one can define a function $K_{\rho}(g)$ ( $K$ like Kernel) which is bi-invariant under $S U(2)$ :

$$
K_{\rho}(g)=\left\langle\rho 0\left|D^{(0, \rho)}(g)\right| \rho 0\right\rangle
$$

Due to its invariance, it is a function of solely the boost angle of $g$. It is useful in studying $L^{2}$ functions over the upper hyperboloid $\mathcal{H}_{+}$. I merely recall basic relations between these functions. For more details, the lector could read [8, 11, 26]. An explicit expression of the Kernel is

$$
K_{\rho}(x, y)=K_{\rho}\left(x^{-1} y\right)=\frac{\sin \rho \theta}{\rho \sinh \theta}
$$

where $\theta$ is the hyperbolic distance (boost parameter) between $x$ and $y$. One can also check that

$$
\int_{0}^{\infty} \rho^{2} d \rho K_{\rho}(x, y)=2 \pi^{2} \delta_{\mathcal{H}_{+}}(x, y)
$$

where $d \mu(\rho)=\rho^{2} d \rho$ is the Plancherel measure restricted to the simple representation $(n=0, \rho)$. Moreover

$$
\int_{\mathcal{H}_{+}} d y K_{\rho}(x, y) K_{\rho^{\prime}}(y, z)=\frac{\delta\left(\rho-\rho^{\prime}\right)}{\rho^{2}} K_{\rho}(x, z)
$$

which means that the Kernel $K$ is a projection onto the $\rho$ Fourier component on the hyperboloid $\mathcal{H}_{+}$.

\section{References}

[1] Laurent Freidel, Etera R Livine, Spin Networks for Non-Compact Groups, hep-th/0205268

[2] Abhay Ashtekar, Jerzy Lewandowski, Projective Techniques and Functional Integration, J.Math.Phys. 36 (1995) 2170-2191, gr-qc/9411046

[3] Carlo Rovelli, Ashtekar for,ulation of general relativity and loop space nonperturbative quantum gravity: A report, Class. Quant. Grav. 8 (1991) 1613-1676 
[4] Laurent Freidel, Kirill Krasnov, Simple Spin Networks as Feynman Graphs, J.Math.Phys. 41 (2000) 1681-1690, hep-th/9903192

[5] Roberto De Pietri, Laurent Freidel, so(4) Plebanski Action and Relativistic Spin Foam Model, Class.Quant.Grav. 16 (1999) 2187-2196, gr-qc/9804071

[6] John W Barrett, Louis Crane, Relativistic spin networks and quantum gravity, J.Math.Phys. 39 (1998) 3296-3302, gr-qc/9709028

[7] John C Baez, John W Barrett, The Quantum Tetrahedron in 3 and 4 Dimensions, Adv.Theor.Math.Phys. 3 (1999) 815-850, gr-qc/9903060

[8] John W Barrett, Louis Crane, A Lorentzian Signature Model for Quantum General Relativity, Class.Quant.Grav. 17 (2000) 3101-3118, gr-qc/9904025

[9] Alejandro Perez, Carlo Rovelli, Spin foam model for Lorentzian General Relativity, Phys.Rev. D63 (2001) 041501, gr-qc/0009021

[10] Alejandro Perez, Carlo Rovelli, 3+1 spinfoam model of quantum gravity with spacelike and timelike components, Phys.Rev. D64 (2001) 064002, gr-qc/0011037

[11] John C Baez, John W Barrett, Integrability for Relativistic Spin Networks, Class.Quant.Grav. 18 (2001) 4683-4700, gr-qc/0101107

[12] Etera R Livine, Immirzi parameter in the Barrett-Crane model?, gr-qc/0103081

[13] Etera R Livine, Daniele Oriti, Barrett-Crane spin foam model from generalized BF-type action for gravity, Phys.Rev. D65 (2002) 044025, gr-qc/0104043

[14] Etera R Livine, Daniele Oriti, Spin Foam Space-Time as Quantum Causal Set, in preparation

[15] Daniele Oriti, Spacetime geometry from algebra: spin foam models for non-perturbative quantum gravity, Rept.Prog.Phys. 64 (2001) 1489-1544, gr-qc/0106091

[16] Sergei Alexandrov, $S O(4, C)$-covariant Ashtekar-Barbero gravity and the Immirzi parameter, Class.Quant.Grav. 17 (2000) 4255-4268, gr-qc/0005085

[17] Serguei Alexandrov, Dmitri Vassilevich, Area spectrum in Lorentz covariant loop gravity, Phys.Rev. D64 (2001) 044023, gr-qc/0103105

[18] Sergei Alexandrov, On choice of connection in loop quantum gravity, Phys.Rev. D65 (2002) 024011, gr-qc/0107071

[19] Serguei Alexandrov, Hilbert Space for Covariant Loop Quantum Gravity, gr-qc/0201087

[20] Sergei Alexandrov, Etera R Livine, SU(2) Loop Quantum Gravity from Covariant Theory, in preparation

[21] Sören Holst, Barbero's Hamiltonian derived from a generalized Hilbert-Palatini action, Phys.Rev. D53 (1996) 5966-5969, gr-qc/9511026

[22] Nuno Barros e Sa, Hamiltonian analysis of General relativity with the Immirzi parameter, Int.J.Mod.Phys. D10 (2001) 261-272, gr-qc/0006013

[23] Carlo Rovelli, Simone Speziale, Reconcile Planck-scale discreteness and the Lorentz-Fitzgerald contraction, gr-qc/0205108

[24] Thomas Thiemann, Introduction to Modern Canonical Quantum General Relativity, gr-qc/0110034

[25] A. W. Knapp Representation of semi-simple groups, Princeton University press.

[26] W Ruhl, "The Lorentz Group and Harmonic Analysis" (WA Benjamin Inc, New York 1970)

[27] I. M. Gel'fand, R. A. Minlos and Z. Ya. Shapiro, Representations of the rotation and Lorentzi groups and their applications (Pergamon Press, 1963), pages 187-189 